\section{Elucidating the pivotal role of convalescent plasma therapy in critically ill COVID-19 patients: A review}

\author{
Seidu A. Richard, ${ }^{1}$ Sylvanus Kampo, ${ }^{2}$ \\ Maite Esquijarosa Hechavarria ${ }^{2}$ \\ ${ }^{1}$ Department of Medicine, Princefield \\ University, Ho; ${ }^{2}$ Department of \\ Anesthesia and Critical care, School of \\ Medicine, University of Health and \\ Allied Sciences, Ho, Ghana
}

\begin{abstract}
World Health Organization (WHO) declared coronavirus disease (COVID-19) a pandemic in March 2020. Currently almost every country in the world has reported cases with moderate to high mortality rates. The European Union (EU), the United States of America (USA) and the United Kingdom (UK) are the severely affected countries. Nevertheless, the WHO is very much concern about countries with weak health systems. The clinical characteristics of COVID-19 varies extensively, ranging from asymptomatic infections to severe as well as critical pneumonia with high mortality rates in the elderly and patients with co-morbid medical illness. Convalescent Plasma Therapy (CPT) has been successfully used in treating various viral disease outbreaks such as 1918 influenza pneumonia pandemic, poliomyelitis, measles, mumps, Machupo virus, Junin virus, Lassa virus, Ebola etc. High-titer specific antibodies maybe capable of binding to Coronavirus$19(\mathrm{CoV}-19)$ and neutralize the viral particles, inhibit entry to uninfected cells, and trigger potent effector mechanisms such as complement activation as well as phagocytosis. Therefore, in most countries with very weak health systems with no Intensive Care Units (ICUs) or trained ICU physicians, early initiation of CPT for severely COVID19 patients may be rewarding. Therefore, solidarity control trials on CPT for COVID19 patients involving large number of patients are urgently needed.
\end{abstract}

\section{Introduction}

In December 2019 a novel strain of coronavirus, officially named Coronavirus19 (CoV-19) was first isolated from three patients with coronavirus disease and communicated to the Chinese Center for Disease Control and Prevention. ${ }^{1,2}$ COVID-
19 was linked to a cluster of acute respiratory illness cases from Wuhan, China. ${ }^{3}$ The World Health Organization (WHO) declared COVID-19 a pandemic in March $2020 .{ }^{4}$ Currently almost every country in the world has reported cases with high to moderate mortality rates. The European Union (EU), the United States of America (USA) and the United Kingdom (UK) are the severely affected countries. Nevertheless, WHO is very much concern about countries with weak health systems.

The clinical presentation of COVID-19 varies extensively, ranging from asymptomatic infections to severe, including critical pneumonia with extreme mortality rates. ${ }^{3}$ Currently, there are no established antiviral medication for the treatment of COVID-19. Also, no vaccines are available for the prevention of COVID-19. Recently, the USA Food and Drug Administration (FDA) has approved the use of Convalescent Plasma Therapy (CPT) for the treatment of critically ill COVID-19 patients on conditions that doctors get approval over the telephone before initiating treatment. ${ }^{5}$ Nevertheless, the disease continuous to kill many patients even in countries with the most robust health systems, because most of these countries outlook the use of CPT for severe COVID-19 patients at the Intensive Care Units (ICUs). In countries with very weak health systems with no ICUs or trained ICU physicians, early initiation of CPT for high risk COVID-19 patients maybe rewarding as well cost reduction.

\section{Overview of convalescent plasma therapy for viral disease out- breaks}

The use of CPT or immunoglobulins for viral infectious disease date to the 1918 Influenza Pneumonia Pandemic (IPP) (Figure 1). ${ }^{5,6}$ Luke et al. with in a metaanalysis involving eight studies with a total number of 1703 influenza pneumonia patients from 1918-1925 who received an infusion of influenza-convalescent human blood products revealed a pooled complete decline of $21 \%(95 \%$ CI $15-27 ; \mathrm{p}<0.001)$ in the overall crude case mortality rate at low risk of bias. ${ }^{6}$ Also, CPT was used in the past to treat diseases such as poliomyelitis, measles, as well as mumps (Figure 1) during outbreaks similar to COVID-19. ${ }^{5}$ Furthermore, CPT was used to successfully treat patients with Machupo virus infections, which caused the Bolivian Hemorrhagic Fever (BHF) in Bolivia (Figure 1). ${ }^{7}$ Moreover, during Junin virus
Correspondence: Seidu A. Richard, Department of Medicine, Princefield University, P. O. Box MA 128, Ho-Volta Region, Ghana.

Tel.: +233508404595.

E-mail: gbepoo@gmail.com

Key words: COVID-19; CPT; ICU; TCM, WHO.

Conflict of interests: The authors declare no conflict of interest.

Contributions: All authors contributed toward literature search, drafting and critically revision of the paper and agree to be accountable for all aspects of the work

Funding: None.

Received for publication: 15 May 2020.

Accepted for publication: 30 October 2020.

This work is licensed under a Creative Commons Attribution-NonCommercial 4.0 International License (CC BY-NC 4.0).

${ }^{(}{ }^{C}$ Copyright: the Author(s), 2020

Licensee PAGEPress, Italy

Hematology Reports 2020; 12:8630

doi:10.4081/hr.2020.8630

infection in Argentina, CPT was used to efficiently treat patients with the Argentinian Hemorrhagic Fever (AHF) (Figure 1). ${ }^{8,9}$ Similarly, in Nigeria, CPT was successfully used to treat Lassa Fever (LF) during the Lassa virus outbreak (Figure 1). ${ }^{10}$ It is wealth noting that almost these outbreaks occurred in countries with very weak health system at time of the disease outbreaks.

In 2003, during the peak of Severe Acute Respiratory Syndrome-coronavirus 1 (SARS-CoV-1) outbreak in China, CPT was an effective treatment option in severe cases (Figure 1). ${ }^{11-13}$ This treatment modality was the last alternative for the survival rate of patients with SARS-CoV-1 whose illness continuous to worsen notwithstanding treatment with pulsed methylprednisolone. ${ }^{14}$ Cheng et al. in 2005 demonstrated that in their study involving 80 SARS-CoV-1 patients that CPT correlated with better rate of hospital discharge approximately 3 weeks after the onset symptom compared with patients who did not receive CPT. ${ }^{15}$ The long hospital stay could be due to the delay in initiation of treatment because patient where put on conversional therapies before the initiation of CTP. Nevertheless, several studies exhibited a shorter hospital stay as well as lower death rate in patients treated with CPT than patients who were 
not on CPT. ${ }^{11,14}$

Hung et al., in a multicenter, prospective, double-blind, randomized controlled trial involving 20 patients in 2009 , demonstrated that convalescent plasma obtained from patients who recovered from influenza A H1N1pdm09 virus infection was an effective and efficient treatment modality for severe influenza A (H1N1) infection (Figure 1). ${ }^{16}$ They indicated that CPT correlated well with a lower viral load, serum cytokine response as well as a decreased death rate within 5 days of symptom onset. ${ }^{16}$ In 2014, WHO approved CPT as one the critical treatment option for patients with Ebola. The use of convalescent plasma obtained from patients who had recovered from Ebola virus disease was efficient and effective practical treatment during these outbreaks (Figure 1). ${ }^{17}$

In 2015, during the Middle East Respiratory Syndrome Coronavirus (MERS-CoV) outbreak, CPT was effective and efficient in treating severe patients (Figure 1). ${ }^{18}$ Based on the success story of CPT, a protocol for it use was established. ${ }^{18}$ Nevertheless, a practicality interventional study involving CPT during MERS-CoV infection treatment failed to detect acceptable high-titer plasma from patients with confirmed or suspected MERS, their close family members, or healthcare workers exposed to MERS. ${ }^{19}$

Mair-Jenkins et al. demonstrated in a meta-analysis that the viral load and death rate was low in patients with severe SARS$\mathrm{CoV}$ and MERS-CoV infections who received various doses of convalescent plasma. ${ }^{20}$ The study further revealed that, CPT was associated with no adverse events or complications. ${ }^{20}$ It is very highly anticipated that, CPT may be a game changer in the fight against COVID-19 most especially in countries with very weak health systems without ICUs or ICU physicians. Thus, control trials are urgently needed to establish the potency of CPT for COVID-19.

\section{Clinical characteristics and potential treatment regimes}

The clinical characteristics of COVID19 varies extensively, ranging from asymptomatic infections to severe, including critical pneumonia with high mortality rates in the elderly and patients with co-morbid medical illness. ${ }^{3}$ Chen et al. observed symptoms such as fever, cough, fatigue, anorexia, myalgia, and diarrhea. ${ }^{3}$ They indicated that these symptomatology's did not differ significantly between dead patients and healed patients. Nevertheless, they observed that dyspnea, chest tightness, as well as disorders of consciousness were more frequent the deceased. ${ }^{3}$ The above symptoms could be indicative for the use of CPT in countries without ICUs. Huang et al indicated that, limited number of patients with COVID-19 had noticeable upper respiratory tract signs and symptoms such as rhinorrhoea, sneezing, or sore throat. ${ }^{21}$ They concluded that the target cells might be located in the lower airway since majority of their patients presented with low respiratory signs and symptoms. ${ }^{21}$

A body of evidence indicates that most dead patients had tachycardia and/or tachypnoea with lower pulse oxygen saturation $(\leq 93 \%))^{3}$ The study further indicated that, in most of the deceased patients, chronic hypertension as well as other cardiovascular comorbidities accounted for mortality compared to recovered patients. ${ }^{3}$ Furthermore, hypoxemia related symptoms were more frequent in patients who eventually died as compared with recovered patients. Patient who eventually died usually developed systematic inflammation as well as multi-organ failure as compared to recovered patients. ${ }^{3}$ The above symptoma- tology point to the fact that patients who died from COVID-19 often aggravates into severe or critical condition on admission before dying. ${ }^{3}$ It is worth noting that the onset of certain symptoms may aid physicians detect patients at risk of a poor outcome and hence early initial of CPT. ${ }^{3}$

Huang et al. indicated that most patients presented with bilateral ground-glass opacities on chest CT scans. ${ }^{21}$ Countries with weak health systems do not have CT scans for the evaluation of patients with COVID19. These countries only have X-Rays for the diagnosis of respiratory diseases. Thus, urgent X-Ray characteristics features of COVID-19 are needed to aid in the diagnosis. These clinical parameters maybe helpfully to frontlines care gives for COVID-19 patients in arriving at optimum decision for the use of CPT for patients in countries with weak health systems. The current treatment of COVID-19 involves $>85 \%$ of anti-viral agents, such as oral oseltamivir $75 \mathrm{mg}$ every $12 \mathrm{~h}$, intravenous ganciclovir $0.25 \mathrm{~g}$ every $12 \mathrm{~h}$, as well as oral lopinavir/ritonavir tablets 400/100mg twice daily. ${ }^{19,22}$ Also, Remdesivir is another promising anti-viral medication under clinical trials. ${ }^{19,22}$ An old

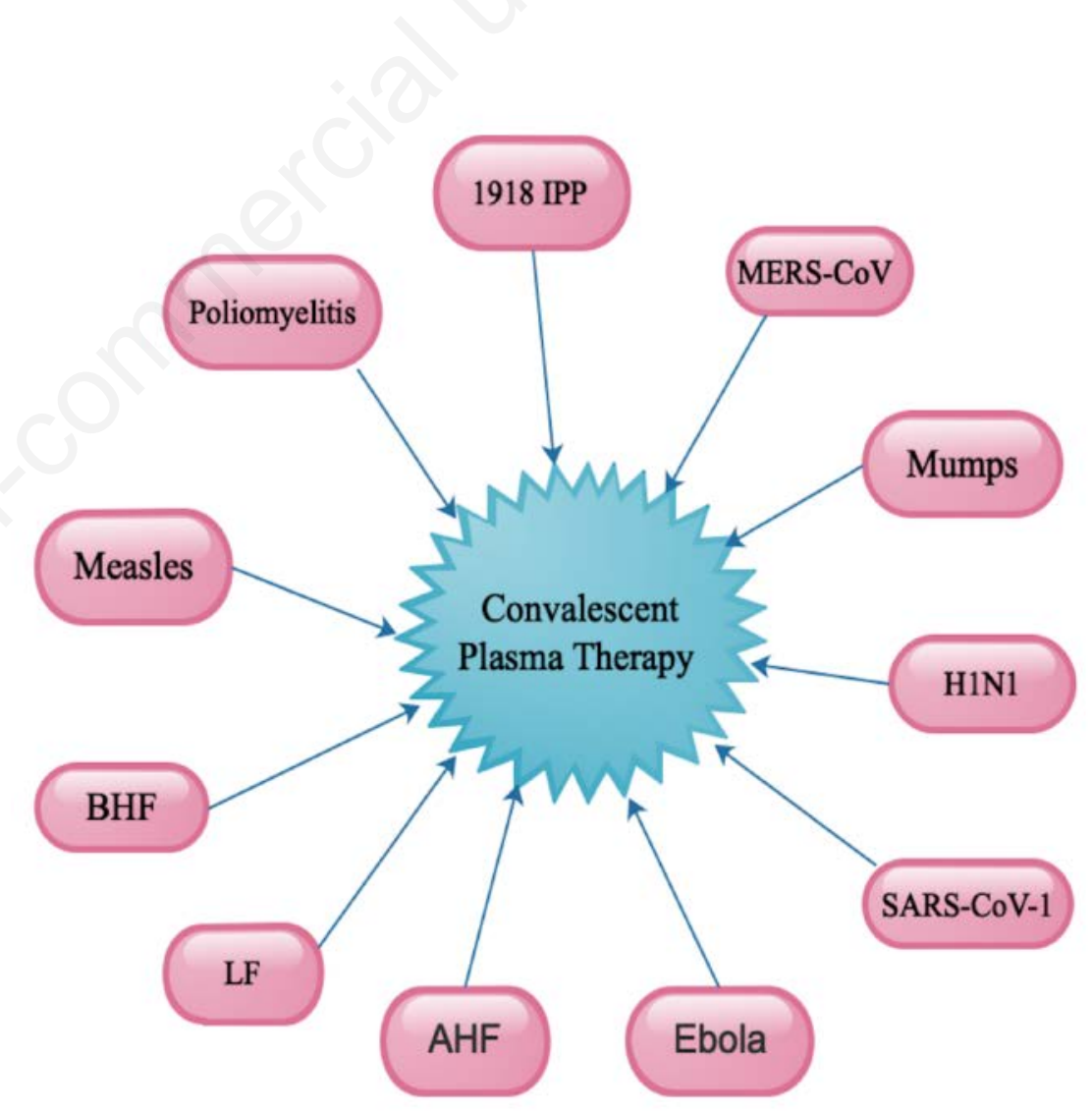

Figure 1. An illustration showing the various disease outbreaks and the use of Convalescent Plasma Therapy (CPT). 1918 influenza pneumonia pandemic = IPP, Argentinian hemorrhagic fever $=$ AHF, Bolivian hemorrhagic fever $=$ BHF, Influenza A = HiN1, Lassa fever = LF, Middle East respiratory syndrome coronavirus = MERS-CoV, Severe acute respiratory syndrome-coronavirus 1 = SARS-CoV-1. 
anti-malarial, chloroquine phosphate or hydroxychloroquine is a derivative of chloroquine, and is effective in inhibiting COVID-19 cellular invasion and thus inhibits exacerbation of pneumonia as well as lung inflammation. ${ }^{19,23}$ Hydroxychloroquine has been approved by the USA FDA for the treatment of COVID-19.

Based on the mechanism of action of hydroxychloroquine, we stipulate it will be best preventive medication as a post-exposion prophylaxis as well as treatment for asymptomatic carries who are likely to spread the virus widely. Clinical trials are urgently needed in this direction. It has been stipulated that $80-90 \%$ of medication used in treating COVID 19 in China were Traditional Chinese Medicine (TCM). ${ }^{19,24}$ Glycyrrhizae Radix Et Rhizoma (Gancao), Astragali Radix (Huangqi), Atractylodis Macrocephalae Rhizoma (Baizhu), Saposhnikoviae Radix (Fangfeng), as well as Lonicerae Japonicae Flo were the five most commonly used herbs. ${ }^{19,24}$

\section{Intensive care management}

The elderly patients ( $>60$ years), male sex, as well as comorbidities like hypertension, diabetes and immunosuppression are linked to higher morbidity and mortality in COVID-19 infection. ${ }^{3}$ These categories of patients are candidates for ICU as well as CPT. Murthy et al. indicated that ICU is very likely to have a substantial influence on patient outcomes. ${ }^{25} \mathrm{~A}$ body of evidence indicates that the death rate appears to be around $2 \%$, and about $25.9 \%$ of the patients with COVID-19 pneumonia required ICU admission, mostly because $20.1 \%$ often develop Acute Respiratory Distress Syndrome (ARDS). ${ }^{19,22}$

There is currently no vaccine or approved anti-viral regime for the treatment of critically ill patients. ICU regimes essentially emphases on supportive care such as oxygenation, ventilation, as well as fluid management. ${ }^{14,19}$ Severe COVID-19 patients may require extracorporeal membrane oxygenation which is usually managed by well-trained ICU doctors. ${ }^{14,19}$ Low income countries do not have these kinds of doctors. Studies have demonstrated that a combination of low-dose systematic corticosteroids, anti-virals and atomized inhalation of interferon have been incorporated in the regime of critical COVID-19 patients. ${ }^{19,26}$ Nevertheless, control trails on the usage of systematic corticosteroids anti-virals and atomized inhalation of interferon for treatment of COVID-19 is urgently needed.

\section{Criteria for convalescent plasma therapy and Mode of adminis- tration}

We advocate that CPT may be beneficial for critical ill COVID-19 patients in countries without ICUs or ICU physicians. In the current COVID-19 outbreak, severe disease is defined as dyspnoea, respiratory frequency 330 breaths per minute, blood oxygen saturation $\leq 93 \%$, ratio of arterial partial pressure of oxygen to fraction of inspired oxygen $\left(\mathrm{PaO}_{2} / \mathrm{FiO}_{2}\right)<300$, or lung infiltrates $>50 \%$ within 24 to 48 hours. ${ }^{5}$ Life threatening disease is defined as respiratory failure, septic shock, or multiple organ dysfunction or failure. ${ }^{5}$ Most of these parameters are measured by the ventilator which are not forecoming in countries with weak health systems. Therefore, clinical parameter will be the critical determiner of CPT regime.

Eligible plasma donors are patients who have recovered from COVID-19 infection. Appropriate protocols such as written informed consent ought to be obtained from eligible patients. ${ }^{4}$ It is advocated that donors should also have tested negative for COVID-19 and other potential viruses such as hepatitis B virus, hepatitis C virus, Human Immune Deficiency Virus (HIV), as well as bacteria like syphilis at the time of blood donation. ${ }^{4}$ Also, laboratory investigation such as white blood cell count, lymphocyte count, chemistry parameter for the dictation of liver and kidney function, Cycle Threshold value $(\mathrm{Ct})$ as well as inflammatory factors like CReactive Protein (CRP), procalcitonin, and IL-6, and serum antibody titer for IgG, IgM, and neutralizing antibodies need to assessed. ${ }^{4}$ In most studies as well as protocols in various viral outbreaks, patients often received a single transfusion of convalescent plasma as the desired therapy(12, 13, 27-29). ${ }^{12,13,27-29}$

The preparation of CPT most strictly meets the WHO criteria for blood and blood products. ${ }^{30-32}$ ABO blood types of the critical ill COVID-19 patients must strictly be compatible with the convalescent plasma donor. ${ }^{4}$ Shen et al. advocates that the patients should be transfused 2 consecutive times with $200-250 \mathrm{~mL}$ of ABO-compatible convalescent plasma. ${ }^{4}$ In all a maximum of $400 \mathrm{~mL}$ of convalescent plasma ought to be transfused on the same day it was obtained from the donor. ${ }^{4}$ We advocate that all other plasma transfusion protocol should be strictly followed to avoid advice transfusion reactions.

\section{Antibodies detection in COVID- 19 patients}

Long et al. demonstrated acute antibody responses to $\mathrm{CoV}-19$ in 285 patients with COVID-19 using a Magnetic Chemiluminescence Enzyme Immunoassay (MCLIA) for virus-specific antibody detection. ${ }^{33}$ They indicated that, the percentage of patients with positive $\mathrm{CoV}-19$-specific IgG was $100 \%$ from day $17-19$ after the onset of symptom while the percentage of patients with positive CoV-19 -specific IgM plateaued at $94.1 \%$ on day $20-22$ after the onset of symptoms. ${ }^{33}$ They further indicated that, $\operatorname{IgG}$ as well as IgM titers were more elevated in severe group than the mild group. ${ }^{33}$ Nevertheless, they observed substantial difference in $\mathrm{IgG}$ titer 2-week after the unset of symptoms. ${ }^{33}$ Their study failed to establish how long the IgG and IgM titers last after the patient with COVID-19 has fully recovered. Their study was unable test samples for virus neutralization and thus the neutralizing actions of the detected IgG antibodies are unknown. Furthermore, they were unable to determine the association between antibody response as well as clinical outcomes and thereafter.

Long et al. further indicated that serum from patients with COVID-19 exhibited no cross-binding to the $\mathrm{S} 1$ subunit of the $\mathrm{CoV}$ 19 spike antigen. ${ }^{33}$ They also did not observe cross-reactivity of serum samples from patients with COVID-19 to nucleocapsid antigens of CoV-19. ${ }^{33}$ Long et al. study however failed to explicitly explain the critical role of IgG as well as IgM in the patients with COVID-19 other than diagnosis of the infection. ${ }^{33}$ Their study did not explore the role of these antibodies in convalescent therapy. Thus, similar studies are warrant to affirm their finding on $\mathrm{CoV}-19$ spike proteins and $\operatorname{IgG}$ and $\operatorname{IgM}$ in the patients with COVID-19. Studies have shown that, the function and viral structure of $\mathrm{CoV}-19$ are modulated by the Envelope protein (E), Membrane protein (M), the Spike protein (S) and the Nucleocapsid protein $(\mathrm{N}) .{ }^{34,35}$ The $\mathrm{N}$ protein aid in the development of the viral capsid as well as arrangement of whole viral structure while the $\mathrm{S}$ protein aid in viral attachment to the host cell via the ACE2 and TMPRSS2. ${ }^{36} \mathrm{We}$ are not convinced that antibodies generated by $\mathrm{CoV}-19$ did not cross-bind with its binding receptors or proteins just like other viral infections.

Okba et al. outlined the elaboration of serologic assays for identification of virus neutralizing antibodies and antibodies to the $\mathrm{N}$ protein as well as various $\mathrm{S}$ protein domains, such as the S1 subunit, and the 
Receptor-Binding Domain (RBD) of CoV19 in an ELISA design. ${ }^{37}$ They assessed CoV-19-specific antibody reactions in severe and mild patients by using serum samples obtained at different times postonset of illness from 3 PCR-established COVID-19 patients from France. ${ }^{37}$ They further observed that, amongst the $\mathrm{S}$ antigens investigated, $\mathrm{S} 1$ was more specific than S2 in detecting CoV-19 antibodies. ${ }^{37}$ The also observed a high degree of conservation in the coronavirus $\mathrm{S} 2$ subunit in comparison to $\mathrm{S} 1 .^{37}$ The detected seroconversion of $\mathrm{IgG}$ during a second week of COVID-19. ${ }^{37}$ In their three in-house ELISAs investigation, the RBD as well as N protein ELISAs were more sensitive than S1 ELISA in identifying antibodies in mildly infected patients and demonstrated greater associations with $\mathrm{PRNT}_{50}$ titers. ${ }^{37}$

A preprint published by $\mathrm{Wu}$ et al. using a pseudotyped-lentiviral-vector-based neutralization assay to measure Cov-19-specific NAbs in plasma obtained from 175 recovered COVID-19 patients with mild symptoms showed higher titers of CoV-19 Receptor Binding Domain (RBD), S1, and S2-specific binding antibodies. ${ }^{38}$ Their stud revealed that, the titers of NAbs plateaued from day 10-15 after the onset COVID-19 and remained stable thereafter in patients. ${ }^{38}$ They also observed that, the NAbs titers in patients correlated with their ages. ${ }^{38}$ Elderly patients had expressively higher titers of NAbs than younger patients. ${ }^{38}$ They concluded that, antibodies targeting on distinctive domains of S protein, such as S1, RBD, and S2, may all contribute to the neutralization. ${ }^{38}$ Their study was limited because, the kinetics of viral loads were not measured and patients in severe as well as critical condition were excluded from their study because they received passive antibody treatment before sample collection. ${ }^{38}$

Zhao et al. explored the dynamics of total Antibody (Ab), $\operatorname{IgM}$ as well as $\operatorname{IgG}$ antibody against Kindly fuse this linse as one. CoV-19 in serial blood samples obtained from 173 patients with established COVID-19. ${ }^{39}$ They used enzyme linked immunosorbent assay (ELISA) to evaluate the antibodies. ${ }^{39}$

The ELISA for absolute antibodies recognition was developed based on double-antigens sandwich immunoassay (AbELISA), utilizing mammalian cell secreted recombinant antigens possessing the Receptor Binding Domain (RBD) of the spike protein of CoV-19 as the immobilized as well as HRP-conjugated antigen. ${ }^{39}$ They detected that, the seroconversion rate of $\mathrm{Ab}$, IgM and $\operatorname{IgG}$ were $93.1 \%$ (161/173), 82.7\% $(143 / 173)$ and $64.7 \%(112 / 173)$, respectively. ${ }^{39}$ They observed that the cumulative seroconversion rate for $\mathrm{Ab}$ and $\mathrm{IgM}$ plateaued at $100 \%$ approximately 1-month after onset of the COVID-19. ${ }^{39}$ They also observe a strong positive correlation between clinical severity and $\mathrm{Ab}$ titer since 2-week after illness onset, for the first time in COVID-19 patients. $^{39}$

Several studies have demonstrated that, in CoVs, the $\mathrm{N}$ protein has high immunogenic activity and is copiously secreted during infection. ${ }^{40-43}$ Grifoni et al. identified five domains on the S glycoprotein of CoV19 predicted to be linked with a robust immune activity. ${ }^{44}$ Liu et al. demonstrated recombinant S-based ELISA was more sensitive than the recombinant N-based one in the detection of $\operatorname{IgM}$ antibodies when they performed a recombinant $\mathrm{N}$-based as well as recombinant S-based ELISAs to detect $\operatorname{IgM}$ and $\operatorname{IgG}$ antibodies in human serum against CoV-19. ${ }^{40}$ This mean that convalescent plasm containing $\operatorname{IgM}$ and $\operatorname{IgG}$ are likely to bind to both S-spike protein and the $\mathrm{N}$ protein leading to lessen of viral load and disease burden (Figure 2).

\section{Convalescent plasma therapy for COVID-19}

A body of evidence indicates that the efficacy and efficiency of CPT is founded on the idea that antibodies from convalescent plasma might suppress viremia. ${ }^{4,14}$ It is demonstrated that the donor plasma has perceptible IgG as well as IgM anti-COVID-19 antibodies and neutralized the virus in in vitro cultures (Figure 2). ${ }^{45}$ Zhen et al. in study involving 21 recovered COVID-19 patients demonstrated that, CPT can discontinue CoV-19 shedding but cannot reduce mortality in critically end-stage COVID-19 patients, therefore, CPT ought to initiated earlier in the course of the disease to achieve favorable outcomes. ${ }^{46}$ The key mechanism via which these immunoglobins bind to COVID-19 antibodies to elicit the desired effect still rigorous investigations. It was observed that, the patients typically develop a primary immune response by days $10-14$, subsequent to virus clearance. ${ }^{15}$

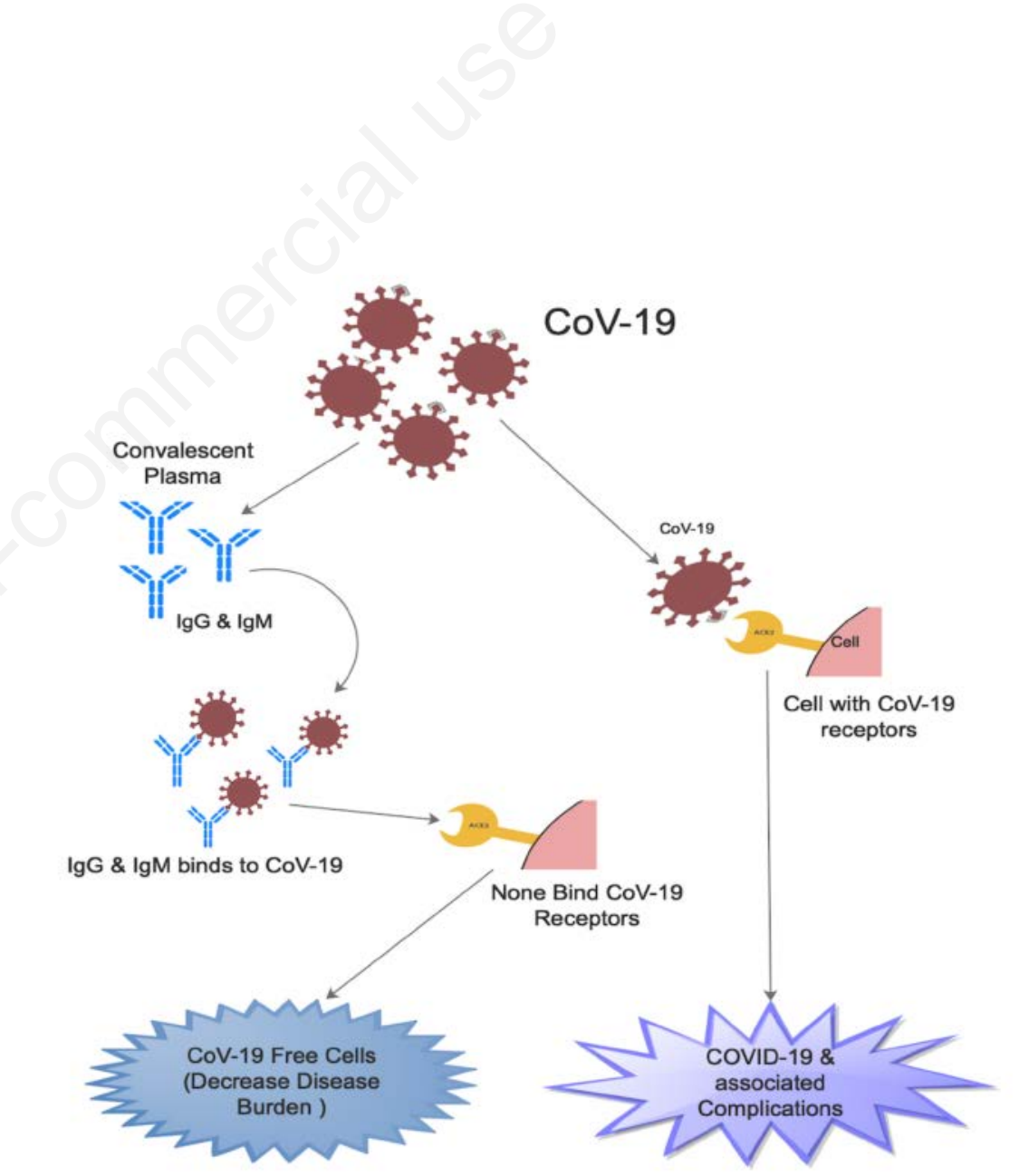

Figure 2. An illustration showing the mechanism via which the IgM and IgG from Convalescent plasma binds to coronavirus-19)(CoV-19) resulting in none binding of CoV-19 receptors as well as decrease coronavirus disease-19 (COVID-19). 
Poh et al. identification of two (S14 and S21) immunodominant linear B-cell epitopes on the $\mathrm{S}$ glycoprotein of $\mathrm{CoV}-19$ that are critical in controlling infection (Figure 2). ${ }^{47}$ They screened 25 convalescence serum samples at 1:1000 dilution for neutralizing antibodies against a pseudo-typed lentivirus secreting CoV-19 S glycoprotein tagged with a luciferase reporter during current COVID-19 pandemic in Singapore. ${ }^{47}$ Six convalescents out of the 25 patients exhibited a good neutralizing response and were used for functional characterization above. ${ }^{47}$ Furthermore, research should also focus on how long immunity last during the convalescent period. In countries with weak health systems, it should be more effective to administer the convalescent plasma at the early stage of disease. ${ }^{15}$

\section{Conclusions}

High-titer specific antibodies maybe capable of binding to COVID-19 and neutralize the viral particles, inhibit entry to uninfected cells, and trigger potent effector mechanisms such as complement activation as well as phagocytosis. The disease continues to kill many patients, even in country with most robust health system, because most of these country outlook CPT for severe COVID-19 at the ICUs. Therefore, in countries with very weak health systems, with no ICUs or trained ICU physicians, early initiation of CPT for severely COVID19 may be rewarding. Therefore, solidarity control trials on plasma therapy for COVID-19 involving large number of patients are urgently needed.

\section{References}

1. Zhu N, Zhang D, Wang W, et al. China Novel Coronavirus Investigating and Research Team. A novel coronavirus from patients with pneumonia in China, 2019. N Engl J Med 2020;382:727-33.

2. Gralinski LE, Menachery VD. Return of the Coronavirus: 2019-nCoV. Viruses 2020;12:135.

3. Chen T, Wu D, Chen H, et al. Clinical characteristics of 113 deceased patients with coronavirus disease 2019: retrospective study. BMJ 2020;368.

4. Shen C, Wang Z, Zhao F, et al. Treatment of 5 Critically Ill Patients With COVID-19 With Convalescent Plasma. JAMA 2020;323:1582-9.

5. Tanne JH. Covid-19: FDA approves use of convalescent plasma to treat critically ill patients. BMJ 2020;368:m1256.
6. Luke TC, Kilbane EM, Jackson JL, Hoffman SL. Meta-analysis: convalescent blood products for Spanish influenza pneumonia: a future $\mathrm{H} 5 \mathrm{~N} 1$ treatment? Ann Internal Med 2006;145:599609.

7. Stinebaugh BJ, Schloeder FX, Johnson $\mathrm{KM}$, et al. Bolivian hemorrhagic fever: a report of four cases. Am J Med 1966;40:217-30.

8. Enria DA, Briggiler AM, Sánchez Z. Treatment of Argentine hemorrhagic fever. Antiviral Res 2008;78:132-9.

9. Ruggiero H, Pérez FI, Milani H, et al. Treatment of Argentine hemorrhagic fever with convalescent's plasma. 4433 cases. Presse Med 1986;15:2239-42.

10. Frame JD, Verbrugge GP, Gill R, Pinneo L. The use of Lassa fever convalescent plasma in Nigeria. Trans R Soc Trop Med Hyg 1984;78:319-24.

11. Soo Y, Cheng Y, Wong R, et al. Retrospective comparison of convalescent plasma with continuing high-dose methylprednisolone treatment in SARS patients. Clin Microbiol Infection 2004;10:676-8.

12. Burnouf T, Radosevich M. Treatment of severe acute respiratory syndrome with convalescent plasma. Hong Kong Med J 2003;9:309.

13. Wong V, Dai D, Wu A, Sung J. Treatment of severe acute respiratory syndrome with convalescent plasma. Hong Kong Med J 2003;9:199-201.

14. Chen L, Xiong J, Bao L, Shi Y. Convalescent plasma as a potential therapy for COVID-19. Lancet Infect Dis 2020;20:398-400.

15. Cheng Y, Wong R, Soo Y, et al. Use of convalescent plasma therapy in SARS patients in Hong Kong. Eur J Clin Microbiol Infect Dis 2005;24:44-6.

16. Hung IF, To KK, Lee C-K, et al. Hyperimmune IV immunoglobulin treatment: a multicenter double-blind randomized controlled trial for patients with severe 2009 influenza A (H1N1) infection. Chest 2013;144:464-73.

17. WHO. Use of convalescent whole blood or plasma collected from patients recovered from Ebola virus disease for transfusion, as an empirical treatment during outbreaks: interim guidance for national health authorities and blood transfusion services. World Health Organization; 2014

18. Arabi Y, Balkhy H, Hajeer AH, et al. Feasibility, safety, clinical, and laboratory effects of convalescent plasma therapy for patients with Middle East respiratory syndrome coronavirus infection: a study protocol. Springerplus 2015;4:709.
19. Cunningham AC, Goh HP, Koh D. Treatment of COVID-19: old tricks for new challenges. Crit Care 2020;24:91.

20. Mair-Jenkins J, Saavedra-Campos M, Baillie JK, et al. The effectiveness of convalescent plasma and hyperimmune immunoglobulin for the treatment of severe acute respiratory infections of viral etiology: a systematic review and exploratory meta-analysis. J Infect Dis 2015;211:80-90.

21. Huang C, Wang Y, Li X, et al. Clinical features of patients infected with 2019 novel coronavirus in Wuhan, China. Lancet 2020;395:497-506.

22. Lai C-C, Shih T-P, Ko W-C, et al. Severe acute respiratory syndrome coronavirus 2 (SARS-CoV-2) and corona virus disease-2019 (COVID-19): the epidemic and the challenges. Int $\mathrm{J}$ Antimicrobial Agents 2020:105924.

23. Gao J, Tian Z, Yang X. Breakthrough: Chloroquine phosphate has shown apparent efficacy in treatment of COVID-19 associated pneumonia in clinical studies. Bioscience Trends 2020;14:72-3.

24. Luo H, Tang Q-1, Shang Y-X, et al. Can Chinese medicine be used for prevention of corona virus disease 2019 (COVID-19)? A review of historical classics, research evidence and current prevention programs. Chinese J Integrative Med 2020:26:243-50.

25. Murthy S, Gomersall CD, Fowler RA. Care for critically ill patients with COVID-19. JAMA 2020;323:1400500.

26. Liu Y, Li J, Feng Y. Critical care response to a hospital outbreak of the 2019-nCoV infection in Shenzhen, China. BioMed Central 2020;24:56.

27. Zhou B, Zhong N, Guan Y. Treatment with convalescent plasma for influenza A (H5N1) infection. N Engl J Med 2007;357:1450-1.

28. Hung IF, To KK, Lee C-K, et al. Convalescent plasma treatment reduced mortality in patients with severe pandemic influenza A (H1N1) 2009 virus infection. Clin Infect Dis 2011;52:44756.

29. Yeh K-M, Chiueh T-S, Siu L, et al. Experience of using convalescent plasma for severe acute respiratory syndrome among healthcare workers in a Taiwan hospital. J Antimicrobial Chemother 2005;56:919-22.

30. WHO. The selection and use of essential medicines. Report of the WHO Expert Committee, 2017 (including the 20th WHO model list of essential medicines and the 6th WHO model list of essential medicines for children). 
WHO; 2017.

31. Wirtz VJ, Hogerzeil HV, Gray AL, et al. Essential medicines for universal health coverage. Lancet 2017;389:403-76.

32. WHO. Selection of essential medicines at country level: using the WHO model list of essential medicines to update a national essential medicines list. WHO; 2020.

33. Long Q-X, Liu B-Z, Deng H-J, et al. Antibody responses to SARS-CoV-2 in patients with COVID-19. Nature Med 2020;26:845-8.

34. Vellingiri B, Jayaramayya K, Iyer M, Narayanasamy A, Govindasamy V, Giridharan B, et al. COVID-19: A promising cure for the global panic. Sci Total Environ 2020:138277.

35. Schoeman D, Fielding BC. Coronavirus envelope protein: current knowledge. Virol J 2019;16:69.

36. Walls AC, Park Y-J, Tortorici MA, et al. Structure, function, and antigenicity of the SARS-CoV-2 spike glycoprotein. Cell 2020;181:281-92.

37. Okba NM, Müller MA, Li W, et al. Severe Acute Respiratory Syndrome Coronavirus 2-Specific Antibody Responses in Coronavirus Disease 2019 Patients. Emerging Infect Dis 2020;26.
38. Wu F, Wang A, Liu M, et al. Neutralizing antibody responses to SARS-CoV-2 in a COVID-19 recovered patient cohort and their implications. MedRxiv 2020

39. Zhao J, Yuan Q, Wang H, Liu W, Liao $\mathrm{X}, \mathrm{Su} \mathrm{Y}$, et al. Antibody responses to SARS-CoV-2 in patients of novel coronavirus disease 2019. Clinical Infectious Diseases. 2020: 139479.

40. Liu W, Liu L, Kou G, et al. Evaluation of Nucleocapsid and Spike Proteinbased ELISAs for detecting antibodies against SARS-CoV-2. J Clin Microbiol 2020;58:e00461-20.

41. Che X-Y, Qiu L-W, Pan Y-X, et al. Sensitive and specific monoclonal antibody-based capture enzyme immunoassay for detection of nucleocapsid antigen in sera from patients with severe acute respiratory syndrome. J Clin Microbiol 2004;42:2629-35.

42. Chen S, Lu D, Zhang M, et al. Doubleantigen sandwich ELISA for detection of antibodies to SARS-associated coronavirus in human serum. Eur J Clin Microbiol Infect Dis 2005;24:549-53.

43. Guan M, Chen HY, Foo SY, et al. Recombinant protein-based enzymelinked immunosorbent assay and immunochromatographic tests for detection of immunoglobulin $G$ antibodies to severe acute respiratory syndrome (SARS) coronavirus in SARS patients. Clin Diagn Lab Immunol 2004;11:287-91.

44. Grifoni A, Sidney J, Zhang Y, et al. A sequence homology and bioinformatic approach can predict candidate targets for immune responses to SARS-CoV-2. Cell Host Microbe 2020:27:671-80.

45. Roback JD, Guarner J. Convalescent Plasma to Treat COVID-19: Possibilities and Challenges. JAMA 2020;323:1561-2.

46. Zeng Q-L, Yu Z-J, Gou J-J, et al. Effect of Convalescent Plasma Therapy on Viral Shedding and Survival in COVID19 Patients. J Infect Dis 2020;222:3843.

47. Poh CM, Carissimo G, Bei W, Amrun SN, Lee CY-P, Chee RS-L, et al. Potent neutralizing antibodies in the sera of convalescent COVID-19 patients are directed against conserved linear epitopes on the SARS-CoV-2 spike protein. BioRxiv 2020; 03.30.015461. 